\title{
Looking beyond the physical management of patients with head and neck cancer
}

SADJ July 2021, Vol. 76 No. 6 p379 - p382

LM Sykes ${ }^{1}$, TV Ntseke ${ }^{2}$, PT Nethononda ${ }^{3}$

\begin{abstract}
Cancers of the head and neck region often leave patients with conspicuous defects, as well as limitations in physical and psycho-social functions. Rehabilitation is challenging and can never fully restore the anatomical, physiological, or functional characteristics that have been lost.

Ideally newly diagnosed cancer patients should be managed by a multidisciplinary team of specialists from various allied fields who will be able to educate them and help them decide on the most appropriate and suitable treatment options. Patients must understand the processes, be of possible side effects and comprehend the limitations aware of rehabilitation. At the same time, clinicians may need to consider issues of distributive justice when deciding which patients will receive which resources, especially in financially limited institutions and countries.
\end{abstract}

The biggest challenge to us as clinicians as well as community members is to try and treat all patients holistically and to address both their physical and psychosocial needs. This requires a fervent commitment to action, and that we all stand together and help each other through difficult times.

\section{INTRODUCTION}

Cancers of the head and neck region often leave patients with conspicuous defects, as well as limitations in physical and psycho-social functions. Rehabilitation is challenging and can never fully restore the anatomical, physiological, or functional characteristics that have been lost. Ideally newly diagnosed cancer patients should be managed by a multidisciplinary team of specialists from various allied

Author affiliations:

1. Leanne M Sykes: BSc, BDS, MDent, IRENSA, Dip Forensic Path, Dip ESMEA, Head of Department of Prosthodontics, University of Pretoria, Pretoria, South Africa.

ORCID Number: 0000-0002-2002-6238

2. Tshegofatso V Ntseke: BDT, BDS, Registrar, Department of Prosthodontics, University of Pretoria, Pretoria, South Africa.

3. Portia T Nethononda: BSc, BDS, PDD, MDent (Pros), Specialist Department of Prosthodontics, University of Pretoria, Pretoria, South Africa.

Corresponding author: Leanne M Sykes

Department of Prosthodontics, University of Pretoria,

Pretoria, South Africa.

Email: leanne.sykes@up.ac.za

Author contributions:

1. Leanne M Sykes: Primary author $-60 \%$

2. Tshegofatso V Ntseke: Second author - $20 \%$

3. Portia T Nethononda: Third author $-20 \%$

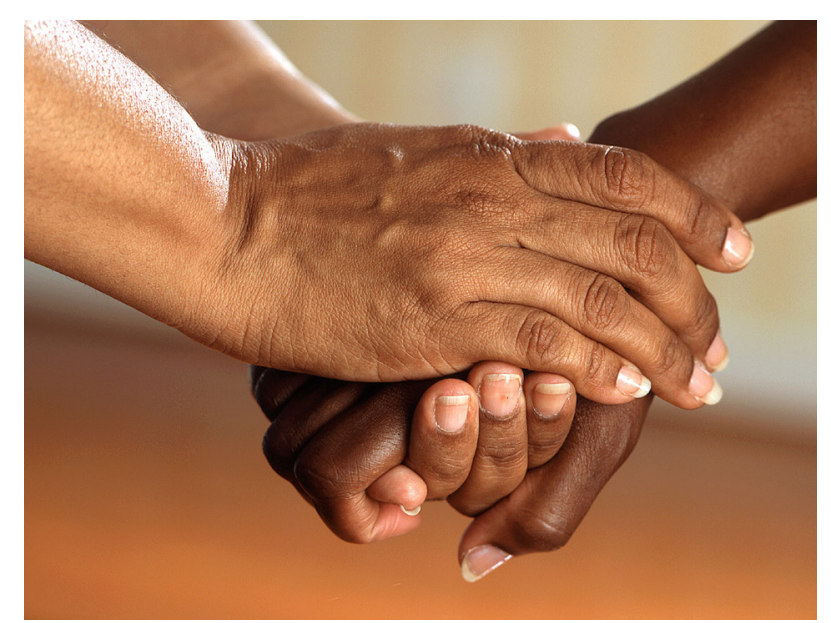

fields who will be able to educate them and help them decide on the most appropriate and suitable treatment options. Patients must understand the processes, be aware of possible side effects and comprehend the limitations of rehabilitation. At the same time, clinicians may need to consider issues of distributive justice when deciding which patients will receive which resources, especially in financially limited institutions and countries.

Challenges faced by newly diagnosed head and neck cancer patients

Many patients who develop head and neck cancer may be unaware of it until it becomes physically noticeable or functionally limiting. Studies have documented a sense of "fatalism about cancer" especially amongst those whose knowledge is based on past experiences of family members or friends. ${ }^{2}$ If the latter only sought treatment in the late stages of their cancer, their outcomes would most likely have been worse, and their ensuing morbidity greater than if it had been treated timeously.

Seeing these poor outcomes in others, may compound the patient's fear and reluctance to seek medical help themselves. They may also delay going to the doctor in the hopes that the cancer will heal itself, out of fear of the unknown, or due to a lack of finances and access to medical facilities. Others may try home remedies or seek help from religious counsellors, alternative therapists, or traditional healers. Patients often look for medical help when the pain, appearance, odour, or functional impediment is too great to handle or conceal. By that time, their tumours may have spread to involve any 
number of surrounding facial areas, requiring far more extensive and invasive means of ablation. The delays in treatment are compounded by long waiting lists and limited staff and facilities at government hospitals.

Initially the patient's presentation, diagnosis and clinical situation should be discussed by a multidisciplinary team of specialists from various allied fields. These include head and neck surgeons, radiotherapists, prosthodontists, plastic surgeons, maxillofacial surgeons, ENT surgeons, audiologists, social workers, dieticians, and psychologists. Treatment may be with surgery, radiotherapy, chemotherapy, or a combination of these modalities, depending on the clinico-pathological and histological diagnosis and tumour site and size.

Patients are usually present in these discussions and may find themselves being examined and discussed by a number of people who tend to talk amongst themselves using technical terms that are difficult to comprehend. The scenario can be scary and intimidating, especially for the newly diagnosed cancer patients, who may already feel as if they have just been handed a death sentence. Their fear is compounded if they can't understand the discussions, which are seldom in layman's terms. In addition, rehabilitation after cancer treatment is a "long process with uncertain outcomes" and not all patients are alerted to this. ${ }^{3}$

They are then expected to make decisions and give consent to treatment without having had the time to fully comprehend the totality of their situation. This cannot be considered ethical for a number of reasons. Their anxiety and duress can impact on their decision-making processes, they may not have been provided with complete or clear education and explanations, and may not have sufficient understanding of the risks and benefits of each option. Autonomy also requires that they be given time to discuss their choices with family members, friends, employers, or other clinicians, in order to get a second opinion. It has been noted that many peoples' health literacy is worse than their general literacy which impacts on their ability to "obtain, process and understand both written and verbal cancer information". They may also struggle to "comprehend information which contains unfamiliar medical vocabulary". ${ }^{2}$ Clinicians may also struggle to quantify the risks, due to the unpredictable nature of cancer and varying patient responses to therapy. Moreover, patients may try to put on a "brave face" infront of family members who have accompanied them, and avoid discussing their situation for fear of upsetting those closest to them.

The patient's treatment choice and decision may be further compromised if one member of the team is more authoritative and biased towards a certain regime. In training institutions patients are generally screened and treated by registrars under the guidance of a consultant. The former are required to fulfil certain procedural quotas during their training time, and may be tempted to "persuade" patients into accepting the treatment modality that fits their needs. Others may be torn by their desire to provide ideal treatment, yet having to settle for carrying out only those procedures that fall within the budgetary constraints of the hospital.
Clinicians may be guilty of adding to the patient's confusion by playing down the severity of the proposed treatment with phrases such as "you have cancer, will you prefer to have it cut out (surgery) or to have the machine (radiotherapy)?" Patients may not be given all the relevant information and could have misperceptions about the implications and side effects of each, yet are expected to make an informed and considered decision almost immediately. For example, if they opt for surgery, they should have an indication of the extent of the resection, if reconstruction will be possible surgically, and if so, how this will be achieved. Will donor sites be involved, will they have limitations in terms of facial appearance and function, the timing and number of operations anticipated, predicted success rates and associated costs. When radiotherapy is an option, they must understand what the procedure will entail, including awareness of the number and timing of sessions that must be strictly adhered to, common side effects such as xerostomia, trismus, limited movement, loss of taste, smell, vision or hearing, mucositis, recurrent oral infections, post radiation caries and the worst scenario, osteoradionecrosis.

Chemotherapy may leave them feeling nauseous, weak, with taste loss or dysgeusia, decreased immunity, and hair loss. Side effects of the latter two are time- and dose-dependent, and may be transient or permanent. Patients also need to know that some tumours may require a combination of surgery, radiotherapy or chemotherapy, and that individual responses and recovery rates are variable and unpredictable.

A further consideration is the harsh reality that in many third world countries and government institutions, waiting lists are prohibitively long, and equipment is not always available or operational. Thus, a patient who needs urgent surgery may only be given a theatre booking months later, by which time the tumour could have spread or become inoperable. Alternatively, one who should be treated with radiotherapy may not receive it if the hospital lacks the appropriate radiation facilities, or if equipment is broken.

These patients will be forced to settle for a less suitable treatment option along with greater adverse side effects. This raises the controversial and debatable issue of distributive justice, and fair allocation of limited resources.

A number of theories have been proposed to help decide on the fairest and most equitable way to share goods and services, in this case health care services and facilities. ${ }^{4}$

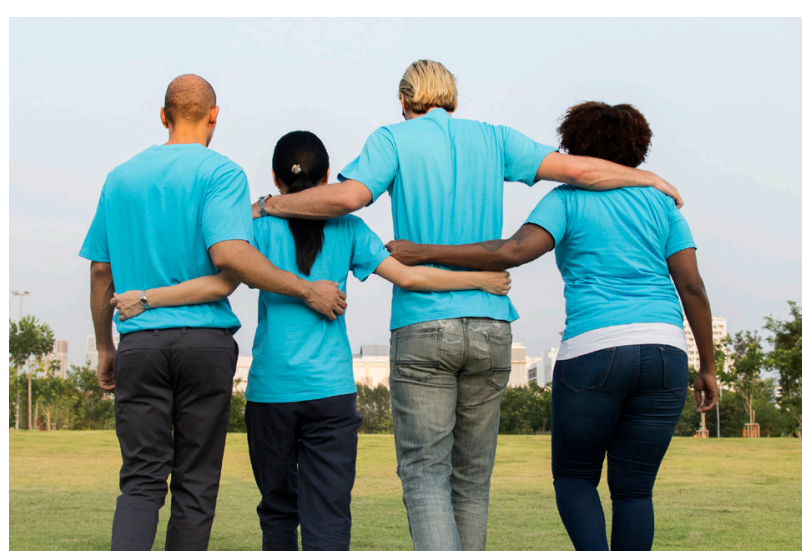


Utilitarian's would advocate sharing resources in a manner that results in the greatest good for the greatest number of people. Libertarianists believe people must get the health care services that they can afford and supports the concept of private health care. In communitarianism the health care needs of the community are given priority over those of individuals, while in egalitarianism everyone would receive an equal distribution regardless of their ability to pay. ${ }^{5}$ In the case of cancer patients, the choice and decision is even more difficult as there are a number of additional considerations such as the age of the patient, their tumour type, stage and prognosis, their ability and willingness to comply with the treatment protocol, the costs of the intervention and subsequent rehabilitation, and their prognosis if they are not given the recommended therapy.

\section{Emotional factors, family counselling and home care}

Facial features and attractiveness "influence the reactions and judgements of other people". ${ }^{3}$ Those considered more attractive are often perceived as being smarter, nicer and more successful than their less attractive counterparts, are received more positively at work and considered more popular in social settings. ${ }^{3}$ Any sudden change in their facial appearance may cause profound psychological difficulties in terms of adaptation, self-acceptance and social adjustment. ${ }^{3}$ In cancer patients, the anxiety and depression may be compounded by the fact that changes are sudden, and the resulting deformities can be patently visible and functionally limiting. They also have to deal with the uncertainty of their prognosis, risks of cancer spread and fear of death.

Some patients develop "marital problems, depression and anxiety, addictions, dysmorphic body images, post-traumatic stress disorder and poor satisfaction with life". ${ }^{6}$ "The patient's personality, ability to cope, amount of social and family support, degree of pain, duration of hospital stay, loss of occupation, anxiety levels, and concern about their physical appearance may all affect their ability to adjust psychologically to their cancer and facial disfigurement". ${ }^{7}$

Home support from both family and trained counsellors is imperative to help integrate them into a normal way of life again. At the same time, throughout their treatment, from the demanding and information loaded consultation session, therapy, healing, rehabilitation, and post treatment monitoring, the focus is primarily on the patient, their treatment, and the outcomes. There is seldom much time or consideration given to the accompanying family members. However, they are an integral part of the team as they will need to provide emotional and/or physical support for the patient in the stressful months and years ahead.

They need to be included in the decision-making process, given advice on what to expect and how to manage the challenges of a debilitated family member, provided with dietary counselling, and introduced to a social worker or psychologist who will be able to "support the supporters". They may also have to take on the unpleasant task of helping patients clean their defects, insert, remove, and clean their prostheses, monitor the area for recurrences and apply necessary medication or dressings. To an inexperienced person this can be upsetting and emotionally disturbing.

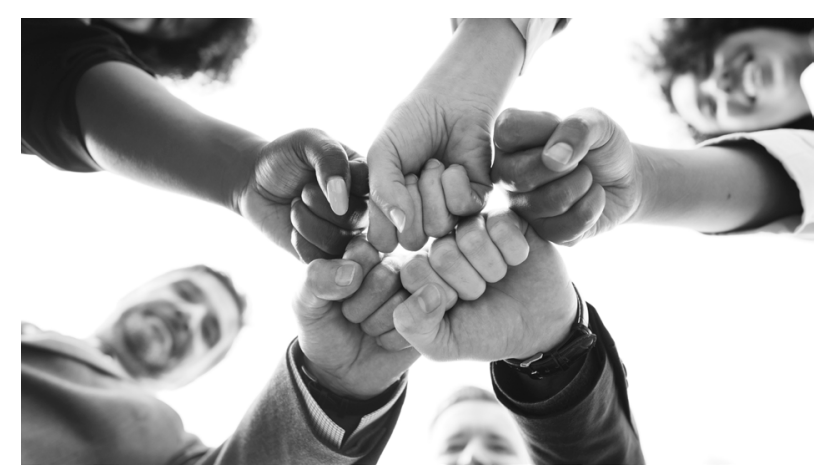

Encouraging and providing physical and emotional support

For patients where surgical reconstruction will not be possible, they need to be advised about the possible prosthetic options, and where they can access these. Ideally the prosthodontist must be part of the multidisciplinary treatment team. This will allow them to make acquaintance with the patient and their families, and provide them with realistic expectation of what is achievable with a prosthesis.

They can provide information that other clinicians assume to be known. For example, a person scheduled to have an orbital enucleation or exenteration must understand that the prosthetic ocular or orbital replacement will never have vision. One who will be fitted with an obturator, or facial prosthesis must know that it will need some form of mechanical retention, that frequent adjustments and replacement are required due to material degradation, and that it will never restore their speech and mastication to pre-operative levels.

The World Health Organisation defines health as "A state of complete physical, mental and social well-being and not merely the absence of disease or infirmity". ${ }^{8}$ This definition makes health depend on "whether the person has established a state of balance within themselves and their environment". 9 It entails that both the patient and their treating doctors remain aware of the need to work together in removing or alleviating the disease as well as help them adapt psychosocially. ${ }^{9}$

Treatment of cancer patients is far too often focused only on the physical eradication of the ailment, and ignores the mental and social aspects of health. It is thus not surprising that head and neck cancer patients hold the highest suicide rates, especially amongst married men. Predictors of suicidal tendencies were self-reported psychiatric histories, substance use as a coping mechanism, reduced quality of life, increased burden of head and neck cancer-related morbidity, pain, speech and masticatory impairment, and psychological distress. ${ }^{10}$

They also reported that medical and pharmacological interventions alone were not enough when it came to treating cancer patients, and stressed the importance of establishing structures within society to address their 
mental state, and strengthen their family relations. They proposed creation of support groups where survivors could meet with those about to go through the process, in order to encourage and assist each another. ${ }^{10}$

\section{CONCLUSION}

When treating patients with head and neck defects, clinicians need to adopt a holistic approach towards establishing if someone is in "good health". They cannot only concern themselves with treatment of the disease and ignore the person suffering with it. They should make it their mission to find out how their patients feel about themselves and their condition, how the illness has affected their lives and that of their families, its impact on their work, and its effects on them psychosocially. They are instrumental role players in helping patients fight the ailment, as well as to live fruitful lives despite the associated side effects. ${ }^{9}$

The biggest challenge to us as clinicians as well as community members is to try and treat all patients holistically and to address both their physical and psychosocial needs. Included in this task is the need to promote health within the community, and to engage the support of those in allied medical disciplines, family members, friends, schools, the media, and even the legislature. This is a challenge which is before us as clinicians and as a community right now, and which requires from us a fervent commitment to action. In this sense it entails that we all stand together and help each other through all of our difficult times.

\section{References}

1. Gilyoma JM, Rambau PF, Masalu N et al. Head and neck cancers: a clinic-pathological profile and management challenges in a resource-limiting setting. BMC Res Notes. 2015; 8: 772 .

2. Davis TC, Williams MV, Marin E, Parker RM et al. Health literacy and cancer communication. A Cancer Journal for Clinicians. 2002; 52(3): 134-49.

3. De Sousa A. Psychological issues in oral and maxillofacial reconstructive surgery. Science Direct. 2008; 46: 661-4.

4. Moodley K and Naidoo S. Ethics and the dental team. Pretoria, Van Schaik publishers. 2010. 38-41.

5. Beauchamp TI, Childress JF. Principles of Biomedical Ethics. $5^{\text {th }}$ Ed. New York: Oxford University Press. 2001.

6. Levine E, Degutis L, Pruzinsky T. et al. Quality of life and facial trauma: psychological and body image effects. Ann Plas Surg. 2005; 54: 502-10.

7. Reed J, Robathan M, Hockenhull M, et al. Children's attitudes towards interacting with peers with different craniofacial anomalies. Cleft Palate Craniofac J. 1999; 36: 441-7.

8. Constitution of the World Health Organization. In: World Health Organization: Basic documents. $45^{\text {th }}$ ed. Geneva: World Health Organization. 2005.

9. Sartorius N. The meanings of health and its promotion. Croat Med J. 2006; 57(4): 662-4.

10. Twigg JA, Anderson JM, Huphris G, et al. Best practice in reducing the suicide risk in head and neck cancer patients: a structured review. British Journal of Oral and Maxillofacial Surgery. 2020; 58: e6-e15. 